\title{
ESCÁNDALOS CORPORATIVOS EN CHILE: IMPACTO EN LOS PRECIOS ACCIONARIOS DE LAS EMPRESAS INVOLUCRADAS*
}

\author{
Vania Donoso, \\ Estudiante de Ingeniería Comercial, Universidad Alberto Hurtado \\ Fernando López ${ }^{* *}$ \\ Profesor de Finanzas, Universidad Alberto Hurtado
}

\begin{abstract}
Resumen
En los últimos años, un grupo de empresas chilenas han protagonizado una serie de escándalos corporativos. Este estudio cuantifica el impacto de estos eventos en los precios accionarios de cinco empresas emblemáticas. Los resultados sugieren que a cuatro semanas del evento, el caso "La Polar" produjo una caída bursátil de 68,4\%; los casos "Colusión de Papel Higiénico" (CMPC) y "Cascadas" (Soquimich) produjeron caídas bursátiles de 13,8\% y 16,2\% respectivamente; mientras que en los casos "Caval" (Banco de Chile) y "Colusión de Pollos" (Cencosud), sus precios accionarios no cayeron de manera significativa. Se presentan hipótesis que podrían explicar estos resultados.
\end{abstract}

\section{La ola reciente de escándalos corporativos en Chile}

En los últimos años, un grupo de empresas chilenas han sido denunciadas por participar en situaciones que van desde lo éticamente cuestionable a situaciones de fraude. Tráfico de influencias, evasión de impuestos, lavado de dinero, soborno, cohecho, prácticas anticompetitivas y falsificación de información contable, son algunas de las situaciones denunciadas (Bonnefoy, 2016). La diversidad de estas denuncias y personas involucradas ha deteriorado la percepción de la ciudadanía respecto al grado de honestidad con que se desarrolla la actividad empresarial en el país. Entre otros efectos, la evidencia internacional sugiere que la falta de confianza pública desincentiva la participación de las personas en los mercados financieros (Guiso, Sapienza \& Zingales, 2004 y 2008; Gianetti \& Wang, 2014), la innovación empresarial (La Porta et al., 1997) y en el desarrollo económico (Knack \& Keefer, 1997; Knack \& Zak, 2001)1.

Las situaciones de fraude corporativo son un fenómeno común en países desarrollados y en vías de desarrollo. A modo de referencia, un estudio reciente muestra que entre 1996 y 2004 una de cada siete grandes empresas estadounidenses estaba involucrada en algún tipo de fraude (Dick, Morse \& Zingales,
2014). Estas empresas destruyeron un $20 \%$ de su valor y el costo de sus fraudes alcanzó los US\$380 mil millones anuales. En el caso chileno, a pesar de que los escándalos corporativos han estado en el centro de la opinión pública, no existen estudios que cuantifiquen el impacto reputacional que han experimentado las empresas involucradas. Este estudio aborda este tema analizando la medida en que estos escándalos afectaron el valor de mercado de un grupo de grandes empresas.

\section{¿Porqué evaluar el impacto en los precios accionarios?}

El análisis de precios accionarios tiene la ventaja de que facilita la medición del costo reputacional asociado a la participación en escándalos corporativos. En efecto, en un mercado en que no hay obstáculos para la transacción de acciones, el precio de una acción representa la disposición de los accionistas a pagar por los flujos de caja que una empresa producirá en el futuro. De esta manera, el precio de la acción incorpora la medida en que un escándalo corporativo afectará a la empresa a través de menores ventas, mayores costos de producción y un mayor costo de capital.

*Este estudio surge a partir de un trabajo de investigación grupal realizado por alumnos del curso de Finanzas Corporativas del programa de MBA de la Universidad Alberto Hurtado dictado en Enero de 2016 por el profesor Fernando López. Se agradece especialmente la contribución de los alumnos Mairim Bermúdez, José Chollet, Héctor Curiche, Álvaro Guzmán, Juan Carlos Lazo, Eduardo López, Rubén Mieses, Maryuli Rodríguez, Felipe Sánchez y Cristián Valdés.

** Email de contacto: felopez@uahurtado.cl

(1) El artículo de López y Reyes (2015) describe con mayor detalle la relación entre confianza pública y desarrollo económico. 


\section{Descripción de los casos considerados}

Para analizar la ola reciente de escándalos corporativos se consideran las siguientes empresas: Banco de Chile (caso Caval), Cencosud (Colusión Pollos), CMPC (Colusión Papel Higiénico), La Polar (caso La Polar) y Soquimich (caso Cascadas) ${ }^{2}$. Estas empresas fueron seleccionadas por dos razones. Primero, al momento del escándalo cotizaban en la bolsa de comercio y eran altamente líquidas. Segundo, son relevantes tanto por su participación en la historia empresarial chilena como por su peso en la economía. En efecto, en 2014 estas empresas explicaban un 16\% del Índice de Precios Selectivo de Acciones (IPSA), su capitalización bursátil ascendió a US\$31 mil millones y sus ventas superaron los US\$29 mil millones, equivalentes a un $12 \%$ del PIB chileno de ese año ${ }^{3}$. El Cuadro 1 presenta una descripción de estos escándalos corporativos y la Figura 1 presenta las fechas en que se hicieron públicos.

\section{CUADRO 1}

\section{ESCÁNDALOS CORPORATIVOS CONSIDERADOS EN ESTE ESTUDIO}

\begin{tabular}{|c|c|}
\hline EMPRESA & DESCRIPCIÓN DEL EVENTO \\
\hline $\begin{array}{l}\text { La Polar } \\
\text { (Caso La Polar) }\end{array}$ & $\begin{array}{l}\text { La empresa fue acusada de repactar créditos de clientes morosos sin su consentimiento en el periodo 2006-2011. La empresa } \\
\text { aumentó sus ingresos por intereses y redujo sus provisiones por créditos incobrables, con lo cual abultaba las utilidades } \\
\text { reportadas. Desde julio de } 2011 \text { la empresa ha sido demandada por el Servicio Nacional del Consumidor (SERNAC) y un grupo } \\
\text { de inversionistas perjudicados. Fuente: Baires, Sullivan \& Chavez (2011). }\end{array}$ \\
\hline $\begin{array}{l}\text { Soquimich } \\
\text { (Caso Cascadas) }\end{array}$ & $\begin{array}{l}\text { En septiembre de } 2013 \text { la Superintendencia de Valores y Seguros (SVS) denunció que el controlador de Soquimich (SQM), } \\
\text { Julio Ponce Lerou, dirigía un esquema de operaciones financieras con las acciones de SQM (y otras empresas bajo su control) } \\
\text { en perjuicio de los accionistas minoritarios. La SVS sostenía que Julio Ponce aprovechó su calidad de máximo accionista de } \\
\text { SQM para vender acciones de ésta a precios inferiores a los de mercado y en condiciones bursátiles que imponían barreras } \\
\text { de acceso a otros inversionistas. Al mismo tiempo, Julio Ponce y sus colaboradores eran controladores de las empresas que } \\
\text { compraban estas acciones y las vendían a precios de mercado, produciendo una ganancia en perjuicio de los accionistas } \\
\text { minoritarios. Cabe señalar que la participación de Julio Ponce en la propiedad de las empresas que compraban acciones de } \\
\text { SQM era mayor a su participación en la propiedad de SQM. De esta manera, sus pérdidas asociadas a la venta de acciones de } \\
\text { SQM a precios inferiores a los de mercado se compensaban con la ganancia asociada a la venta de estas acciones por parte } \\
\text { de sus empresas relacionadas o bajo su control. Fuente: SVS ( } 2013 \text { y 2014) }\end{array}$ \\
\hline $\begin{array}{l}\text { Banco de Chile } \\
\text { (Caso Caval) }\end{array}$ & $\begin{array}{l}\text { En diciembre de } 2013 \text {, el Banco otorgó un crédito a la empresa Caval, de propiedad de la nuera de la actual presidenta } \\
\text { Michelle Bachelet, Natalia Compagnon. Esta operación fue altamente cuestionada por las siguientes razones. Primero, el } \\
\text { proyecto consistía en la compra de tres terrenos de uso agrícola por US\$10,7 millones para luego venderlos en US\$15,6 } \\
\text { millones cuando entrara en vigencia un nuevo plan regulador que permitíía darle un uso urbano a dichos terrenos. Segundo, } \\
\text { el vicepresidente y miembro de la familia controladora del Banco, Andrónico Luksic, se reunió con Natalia Compagnon y su } \\
\text { esposo Sebastián Dávalos, hijo de la presidenta Bachelet. Entre los aspectos que han sido cuestionados de esta reunión se } \\
\text { encuentra el hecho que Andrónico Luksic solicitó expresamente la participación de Sebastián Dávalos y que el vicepresidente } \\
\text { de un banco de esta envergadura generalmente no se involucra en este tipo de proyectos. Tercero, el crédito fue aprobado a } \\
\text { pesar que la empresa Caval tenía un nivel de patrimonio bajo en relación a lo que típicamente aceptan los bancos para ese } \\
\text { tipo de proyectos. En la práctica, la empresa Caval obtuvo el crédito el día } 16 \text { de diciembre de } 2013 \text {, compró los terrenos el } \\
\text { día } 17 \text { de enero de } 2014 \text { y luego los vendió el día } 5 \text { de febrero de } 2015 \text {. Fuente: Sallaberry (2015a \& 2015b) y Collados \& von } \\
\text { Baer (2015). }\end{array}$ \\
\hline $\begin{array}{l}\text { CMPC } \\
\text { (Caso } \\
\text { Papel Higiénico) }\end{array}$ & $\begin{array}{l}\text { Esta empresa fue denunciada en octubre de } 2015 \text { por la Fiscalía Nacional Económica (FNE) por su presunta participación } \\
\text { en un esquema de colusión con sus competidores directos. El esquema operaba a través de la fijación de precios y cuotas } \\
\text { de producción en el mercado del papel higiénico chileno. Las empresas involucradas representaban más del } 90 \% \text { de este } \\
\text { mercado. Según la FNE, los acuerdos operaron entre los años 2000-2011. A la fecha, no existen estimaciones de los perjuicios } \\
\text { ocasionados por estas prácticas. Fuente: FNE (2015) }\end{array}$ \\
\hline $\begin{array}{l}\text { Cencosud } \\
\text { (Caso Colusión } \\
\text { Pollos) }\end{array}$ & $\begin{array}{l}\text { En enero de 2016, la FNE denunció a Cencosud por su presunta participación en un esquema de colusión con las empresas } \\
\text { Walmart y SMU. Este esquema operó entre los años } 2008 \text { y } 2011 \text { a través de la fijación de precio de venta y cuotas de } \\
\text { producción de carne fresca de pollo por parte de los productores. Estas empresas concentran más del } 90 \% \text { de la industria } \\
\text { supermercadista y más de } 90 \% \text { de la venta de pollo fresco del mercado. Fuente: FNE (2016) y Skoknic \& Figueroa (2016a y } \\
2016 \text { b) }\end{array}$ \\
\hline
\end{tabular}

Fuente: Elaboración propia.

(2) La empresa Penta no se considera porque sus acciones no se transan en la bolsa.

(3) Fuente: cifras calculadas sobre la base de información pública de la Bolsa de Comercio de Santiago y el Banco Central de Chile. 


\section{FIGURA 1}

Línea de tiempo de los escándalos corporativos considerados en este estudio

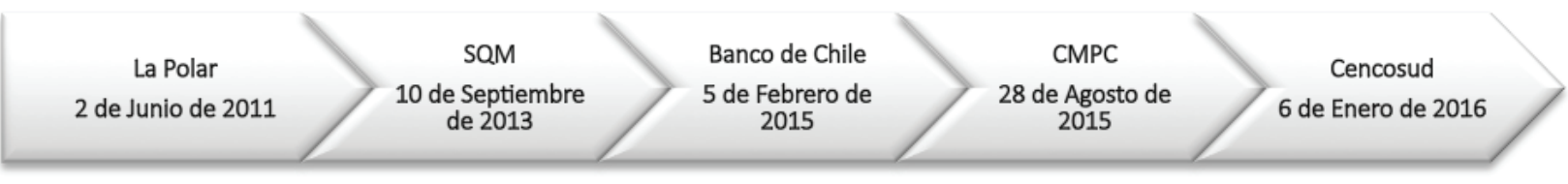

Fuente: Elaboración propia.

\section{Resultados}

El Gráfico 1 presenta la evolución de los precios accionarios entre una y cuatro semanas desde que se hicieron públicos cada uno de los escándalos corporativos considerados. La escala de precios accionarios fue estandarizada de manera que todos fueran iguales a \$100 al momento del escándalo. Se aprecia que la empresa La Polar fue una de las más afectadas, con una caída de $73 \%$ a cuatro semanas del evento. Por su parte, los precios accionarios de CMPC y Soquimich experimentaron caídas de $17,4 \%$ y $14,6 \%$ respectivamente. Por último, no se observan caídas significativas para las empresas Banco de Chile ni Cencosud.

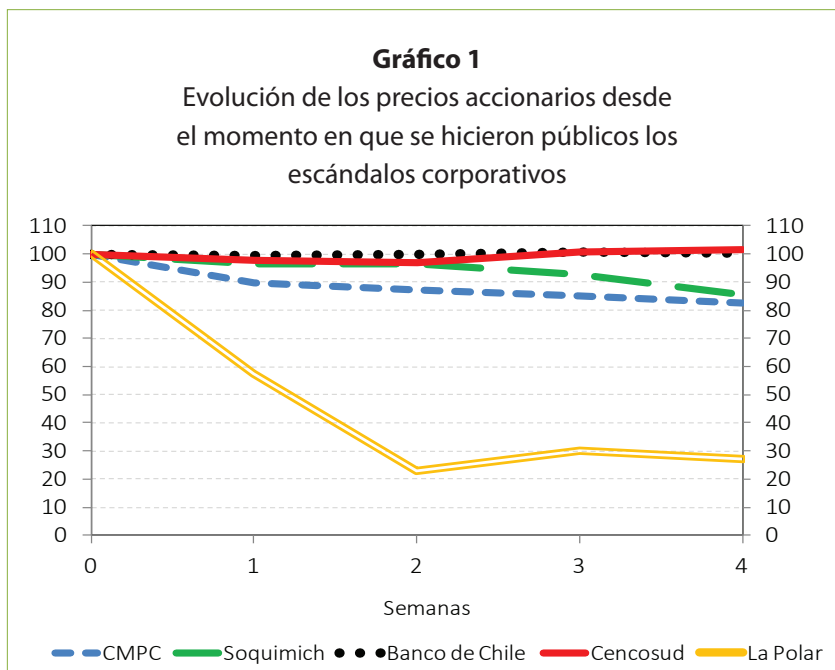

Nota: Los precios accionarios fueron ajustados de manera que sean iguales a $\$ 100$ el día del evento. Los datos fueron obtenidos de la Bolsa de Comercio de Santiago.

Para medir el impacto de los escándalos corporativos en los precios accionarios de las empresas involucradas es necesario comparar la rentabilidad que efectivamente tuvo la acción desde el momento en que se hizo público el escándalo, con la rentabilidad que se hubiese observado en ausencia de éste. Para estos efectos, en primer lugar, es necesario aislar el efecto de las tendencias del mercado bursátil en el precio de la acción durante el periodo analizado. Para ilustrar la importancia de este ajuste, supongamos que la bolsa chilena hubiese experimentado un alza de $5 \%$ a cuatro semanas desde que se hizo público el caso de Colusión del Papel Higiénico (CMPC). Si los precios de las acciones de CMPC, en promedio, se mueven en la misma dirección y magnitud que la bolsa chilena, el impacto del caso hubiese sido igual a $17,4 \%+5 \%=22,4 \%$ y no el $17,4 \%$ que cayó el precio de la acción.

El Gráfico 2 presenta estimaciones de rentabilidad acumulada por sobre la expectativa de mercado a una y cuatro semanas desde que se hicieron públicos los escándalos. Esta rentabilidad se obtiene a partir de la suma de las diferencias entre las rentabilidades observadas y las rentabilidades esperadas para cada acción de acuerdo a la evolución del mercado bursátil. El precio de las acciones de La Polar tuvo una caída de $68,4 \%$ por sobre lo que se hubiese esperado de acuerdo a la evolución del mercado. Los precios de CMPC y Soquimich experimentaron caídas de $13,8 \%$ y $16,2 \%$ que no se explican por las fluctuaciones de mercado. Por último, no se observan caídas significativas en los precios de las acciones de Banco de Chile y Cencosud. Desde un punto de vista cualitativo y cuantitativo se aprecia que los resultados son similares a los reportados en el Gráfico 1.

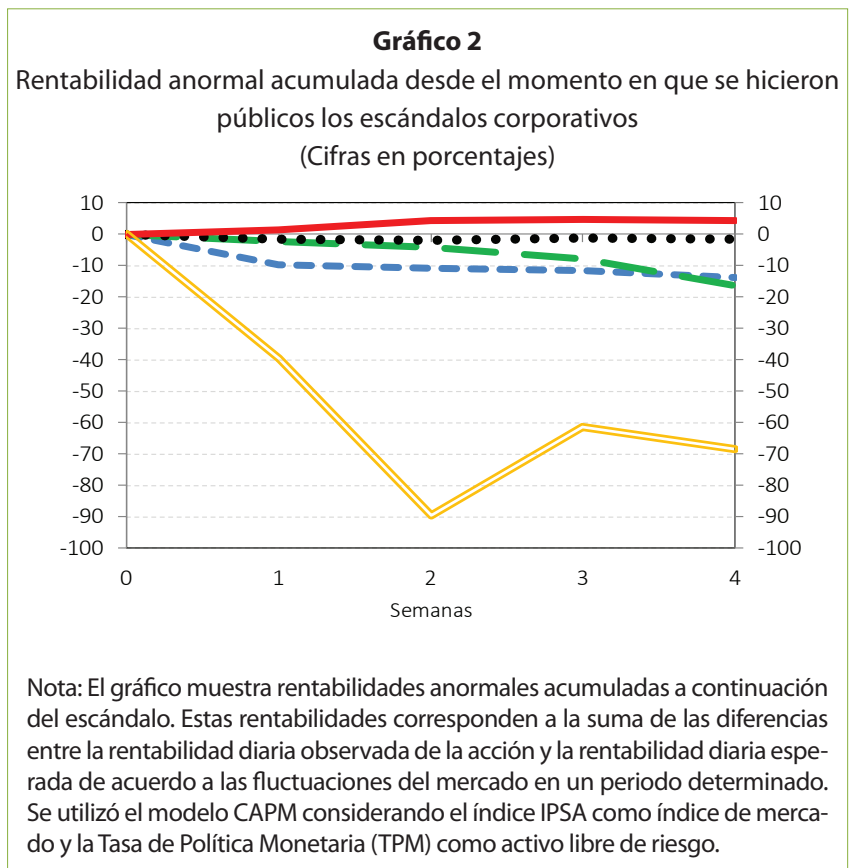


Una segunda posibilidad que es necesario descartar es la presencia de otros eventos que podrían afectar el precio de la acción y que no están relacionados con el escándalo o con fluctuaciones de la bolsa local. Para abordar este punto, se analizaron las noticias relacionadas con cada una de las empresas hasta cuatro semanas después de ocurridos los eventos estudiados. En los casos de La Polar, CMPC, Banco de Chile y Cencosud no se encontraron noticias que pudieran explicar variaciones en el precio de las acciones que no estuvieran relacionadas con el conocimiento público del escándalo. En el caso de Soquimich se observa una caída en el retorno acumulado anormal de $8 \%$ durante la cuarta semana. Una explicación posible es un aumento de capital propuesto por el grupo controlador que fue interpretado por el mercado como una operación que iría en detrimento de los inversionistas minoritarios (Eyzaguirre \& Ohlbaum, 2015). A pesar de que el aumento de capital es un evento independiente del escándalo, lo más probable es que la magnitud de la reacción del mercado se explique por el conocimiento público del caso "Cascadas".

\section{¿Qué factores podrían explicar las diferencias en los resultados?}

Una de las principales características de los resultados anteriores es su heterogeneidad. En un extremo, el precio de la acción de La Polar cayó un $68,4 \%$ por sobre lo que se hubiese esperado de acuerdo a la tendencia del mercado bursátil. A diferencia de los otros casos considerados, este se distinguió por la falsificación de información contable. En efecto, a pocos días de que el caso saliera a la luz pública, la empresa declaró que sus provisiones por créditos incobrables habían sido subestimadas en un monto equivalente a 7 veces las utilidades del año anterior4. Esta cifra no consideraba compensaciones a los consumidores, compensaciones a inversionistas y multas por diversas infracciones a la normativa vigente que surgirían con el conocimiento público del caso.

Los escándalos que involucraron a CMPC y Soquimich tuvieron un impacto menor, cercano al 15\%. En el caso de CMPC, el grueso del impacto podría atribuirse a la expectativa de una menor demanda esperada por su papel higiénico asociada a una sanción social por parte de los consumidores, así como las posibles multas y compensaciones que podrían surgir cuando concluyan los procesos judiciales en que se encuentra involucrada. A diferencia de CMPC, Soquimich vende commodities en los mercados internacionales $y$, por lo tanto, su demanda es poco sensible a este tipo de situaciones. Por su parte, en el caso de Soquimich, el grueso del impacto podría atribuirse a dos factores. Primero, un mayor costo de capital exigido por inversionistas minoritarios que perciben una mayor probabilidad de resultar desfavorecidos en transacciones dirigidas por el grupo controlador. Segundo, la mayor exposición a fiscalización por parte de las autoridades y la eventual detección de otras situaciones que podrían afectar negativamente el precio de la acción.

Por último, en el caso de Banco de Chile y Cencosud no se observan efectos significativos en sus precios bursátiles. En el primer caso, el resultado puede explicarse por tres factores: bajo monto de la operación en relación a los activos del Banco, falta de evidencia suficiente que permitiera comprobar la presencia de algún delito y ausencia de sanción por parte de los inversionistas que podrían haber vendido sus acciones. Por su parte, el resultado para Cencosud es consistente con dos mecanismos que pudieron operar de manera simultánea. Primero, la carne fresca de pollo tiene una participación pequeña dentro de la variedad de productos que vende un supermercado. Segundo, los supermercados involucrados representan más de $90 \%$ de la industria supermercadista $y$, por lo tanto, las personas tienen alternativas limitadas para adquirir este tipo de productos.

\section{Conclusiones}

Recientemente, la opinión pública chilena se ha enterado de que un grupo de empresas han sido protagonistas de una serie de escándalos corporativos. Este artículo presenta un análisis cuantitativo del impacto que estos eventos tuvieron en los precios de las acciones de las empresas involucradas. A cuatro semanas de los escándalos, el conocimiento público del caso "La Polar" estuvo acompañado de una caída bursátil de 68,4\%; los casos "Colusión de Papel Higiénico" (CMPC) y "Cascadas" (Soquimich) estuvieron asociados a caídas bursátiles de 13,8\% y $16,2 \%$ respectivamente, mientras que en los casos "Caval" (Banco de Chile) y "Colusión Pollos" (Cencosud), sus precios accionarios no cayeron. Estos resultados no se explican por las tendencias del mercado bursátil. Tampoco se explican por otras situaciones que pudieron haber afectado los precios accionarios y que no están relacionadas con los eventos analizados. El artículo presenta una serie de hipótesis alternativas que podrían explicar la heterogeneidad de los resultados encontrados.

\section{Referencias}

Baires, R., J. Sullivan \& A. Chavez (8 de julio de 2011), Hitos en una historia de tiempo. La Polar, La historia de una gran estafa. Obtenido de CIPER. Centro de Investigación Periodística: http://ciperchile. $\mathrm{cl} /$ wp-content/uploads/LT-POLAR-RB-ACH-FINAL_2.pdf 
Bonnefoy, P. (7 de julio de 2016). As graft cases in Chile multiply, a 'Gag Law' angers journalists. Obtenido de The New York Times: http://nyti.ms/1S4SPnU

Collados, A. \& A. von Baer (13 de febrero de 2015). Escritura revela que el 5 de febrero se firmó compraventa en la que Caval vendió terrenos. Obtenido de Economía y negocios: http://www.economiaynegocios.cl/noticias/noticias.asp?id=135668

Dick, A., A. Morse \& L. Zingales. (2014). How pervasive is corporate fraud?, working paper.

Eyzaguirre A. \& P. Ohlbaum (5 de marzo de 2014).SVS autoriza aumento de capital de cascadas y AFP analizan si suscribirán operación. Obtenido de Economía y negocios: http://www.economiaynegocios.cl/noticias/noticias.asp?id=117599

FNE (06 de enero de 2016). Fiscalía nacional económica presenta requerimiento por colusión entre las cadenas de supermercados. Obtenido de FNE: http://www.fne.gob.cl/wp-content/uploads/2016/01/requ_01_2016.pdf

FNE (28 de octubre de 2015). Fiscalía nacional económica presenta requerimiento por colusión contra CMPC y SCA. Obtenido de FNE: http://www.fne.gob.cl/2015/10/28/fne-presenta-requerimiento-por-colusion-contra-cmpc-y-sca/

Gianetti, M. \&T. Wang. (Por aparecer). Corporate scandals and household stock market participation, Journal of Finance.

Guiso, L., P. Sapienza, \& L. Zingales. (2004). The role of social capital in financial development. American Economic Review 94, 526-556.

Guiso, L., P. Sapienza, \& L. Zingales. (2008). Trusting the stock market, Journal of Finance 63, 2557-2600.

Knack, S. \& P. Keefer. (1996). Does social capital have an economic payoff?: A cross-country Investigation, Quarterly Journal of Economics, 112 (4), 1251-1288.

La Porta, R., F. Lopez de Silanes, A. Schleifer \& R. Vishny. (1997). Trust in large organizations, American Economic Review 87 (2), 333-338.
López, F. \& R. Reyes. (2015). Confianza pública y desarrollo económico. Observatorio Económico № 93, Universidad Alberto Hurtado. Salaberry, J. (5 de febrero de 2015a). Un negocio Caval. Revista Qué pasa. Obtenido de http://www.quepasa.cl/articulo/actualidad/2015/02/1-16218-9-un-negocio-caval.shtml/

Salaberry, J. (9 de febrero de 2015b). La reunión de Andrónico Luksic con Sebastián Dávalos Bachelet y Natalia Compagnon para gestionar el crédito por UF 266 mil. Obtenido de Revista Qué pasa: http://www.quepasa.cl/articulo/actualidad/2015/02/116220-9-la-reunion-de-andronico-luksic-con-sebastian-davalosbachelet-y-natalia.shtml/

Skoknic. F. \& Figueroa. J. (26 de enero de 2016). Colusión: Los testimonios que pusieron a los supermercados en el banquillo de los acusados. Obtenido de CIPER: http://ciperchile.cl/2016/01/26/ colusion-los-testimonios-que-pusieron-a-los-supermercados-en-el-banquillo-de-los-acusados/

Skoknic. F. \& Figueroa. J. (6 de enero de 2016). Nueva colusión por el precio del pollo. Acusan a las tres principales cadenas de supermercado. Obtenido de CIPER: http://ciperchile.cl/2016/01/06/ nueva-colusion-por-el-precio-del-pollo-acusan-a-las-tres-principales-cadenas-de-supermercados/

SVS (10 de septiembre de 2013). SVS formula cargos en relación a operaciones de sociedades cascadas. Obtenido de http://www. svs.cl/portal/prensa/604/w3-article-13858.html

SVS (2 de septiembre de 2014). SVS sanciona a personas, ejecutivos y corredora de bolsa en el marco de la investigación sobre Sociedades Cascada. Obtenido de sala de prensa SVS: http://www.svs. $\mathrm{cl} /$ portal/prensa/604/w3-article-17480.html

Zak, P. \& S. Knack. (2001). Trust and growth, Economic Journal 111, 295-321. 